\title{
RESONANCE WIDTHS FOR GENERAL HELMHOLTZ RESONATORS WITH STRAIGHT NECK
}

\author{
THOMAS DUYCKAERTS ${ }^{1}$, ALAIN GRIGIS ${ }^{1}$ \& ANDRÉ MARTINEZ ${ }^{2}$
}

\begin{abstract}
We prove an optimal exponential lower bound on the width of the resonance associated to the first eigenvalue of the cavity for a general Helmholtz resonator with straight neck, in any dimension.
\end{abstract}

\section{INTRODUCTION}

A resonator consists of a bounded cavity (the chamber) connected to the exterior by a thin tube (the neck of the chamber). The frequencies of the sounds it produces are determined by the shape of the chamber, while their duration by the length and the width of the neck in a non-obvious way, and our goal is to understand these. Mathematically, this phenomenon is described by the resonances of the Dirichlet Laplacian $-\Delta_{\Omega}$ on the domain $\Omega$ consisting of the union of the chamber, the neck and the exterior.

We recall that resonances are the eigenvalues of a complex deformation of $-\Delta_{\Omega}$; their real and imaginary parts are the frequencies and inverses of the half-lives, respectively, of the corresponding vibrational modes. It is of obvious physical interest to estimate these two quantities as precisely as possible. One practical way to do this involves studying this problem in the asymptotic limit when the width $\varepsilon$ of the neck tends to zero. Those resonances with imaginary parts tending to zero converge to the eigenvalues of the Dirichlet Laplacian on the cavity, and there is an exponentially small upper bound for the absolute values of the imaginary parts (the widths) of the resonances.

Many papers have been devoted to this problem (see, e.g., [Be, $\mathrm{Fe}, \mathrm{HM}$, MN1, MN2], and references therein). For instance, in [Be], it is proved that, as the width of the neck becomes small, these resonances approach either an eigenvalue of the cavity, or a resonance of the exterior region. In [Fe, a construction of states that are concentrated in the cavity is obtained, with lower estimates on their sojourn time (and thus, upper estimates on the widths of resonances, that is, the absolute value of their imaginary parts).

2000 Mathematics Subject Classification. Primary 81Q20 ; Secondary 35P15, 35B34.

Key words and phrases. Helmholtz resonator, scattering resonances, lower bound.

${ }^{1}$ Université Paris 13, Institut Galilée, Département de Mathématiques, avenue J.-B. Clément, 93430 Villetaneuse, France. TD is partially supported by ANR grant SchEq, ERC grant Dispeq and ERC advanced grant BLOWDISOL.

${ }^{2}$ Università di Bologna, Dipartimento di Matematica, Piazza di Porta San Donato 5, 40127 Bologna, Italy. 
In [HM], a very general exponentially small upper bound on the widths of resonances is proved.

However, without very restrictive hypotheses, no lower bound is known. We mention in particular that lower bounds are known in the one-dimensional case [Ha, HaSi]. As for the higher dimensional case, we can mention FL, [Bu, HS, FLM] which contain results concerning exponentially small widths of quantum resonances, but these do not apply to a Helmholtz resonator (in particular, the semiclassical lower bound obtained in [HS] is optimal).

In [MN1, an optimal lower bound has been optioned for very particular two-dimensional Helmholtz resonators, for which the exterior consists of an infinite straight half tube. Then, the result has been extended in MN2 to much more general two-dimensional Helmholtz resonators, under the condition that the neck meets the boundary of the external region perpendicularly to it, and that the exterior region is concave and symmetric there. Moreover, an extension to larger dimensions (up to 12) was obtained, but only for necks with a square section.

Here, we plan to generalize the result to any $n$-dimensional Helmholtz resonator with straight neck, without particular assumption on the section of the neck or on the boundary near the mouth of the neck. (see Theorem 2.2).

As in [MN2], the problem is first related to a lower bound on the resonant function in a large annulus. Assuming, by contradiction, that this function is small there, the smallness can be propagated up to a small neighborhood of the end part of the neck, by means of general Carleman estimates. In order to have a good enough control on the rate of decay near the mouth of the neck, however, we need stronger results. These are furnished to us by the powerful tool of the so-called "limiting Carleman weights" developed in [KSU]. Indeed, they give a framework where more precise Carleman estimates can be done, leading to an exponential decay estimate on the resonant function with a rate going to zero as we approach the neck, but not too quickly. Afterwards, this permits us to propagate the estimate in the inside part of the neck, this time by using explicit Carleman estimates. At that point, the contradiction is obtained as in [MN2, by using a result of [BHM1, BHM2] on the size of the Dirichlet eigenfunctions of the cavity.

\section{Assumptions AND Results}

Let $\mathcal{C}$ and $\mathcal{B}$ be two bounded open sets in $\mathbb{R}^{n}(n \geq 2)$, with $\mathcal{C}^{\infty}$ boundary, and denote by $\overline{\mathcal{C}}, \overline{\mathcal{B}}$ their closures, and by $\partial \mathcal{C}, \partial \mathcal{B}$ their boundaries. We assume that $\mathcal{C}$ is connected, $\mathcal{B}$ is contractible, and that Euclidean coordinates $x=\left(x_{1}, \ldots, x_{n}\right)=:\left(x_{1}, x^{\prime}\right)$ can be chosen in such a way that, for some $L>0$, one has,

$$
\overline{\mathcal{C}} \subset \mathcal{B} \quad ; \quad 0 \in \partial \mathcal{C} \quad ; \quad\left(L, 0_{\mathbb{R}^{n-1}}\right) \in \partial \mathcal{B} \quad ; \quad[0, L] \times\left\{0_{\mathbb{R}^{n-1}}\right\} \subset \overline{\mathcal{B}} \backslash \mathcal{C} .
$$

We also assume,

$$
[0, L] \times\left\{0_{\mathbb{R}^{n-1}}\right\} \text { is transversal to } \partial \mathcal{B} \text { at }\left(L, 0_{\mathbb{R}^{n-1}}\right) \text {, and to } \partial \mathcal{C} \text { at } 0_{\mathbb{R}^{n}} .
$$


Let $D_{1} \subset \mathbb{R}^{n-1}$ be a bounded domain containing the origin, with smooth boundary $\partial D_{1}$. For $\varepsilon>0$ small enough, we set $D_{\varepsilon}:=\varepsilon D_{1}$ and,

$$
\begin{aligned}
& \mathbf{E}:=\mathbb{R}^{n} \backslash \overline{\mathcal{B}} ; \\
& \mathcal{T}(\varepsilon):=\left(\left[-\varepsilon_{0}, L+\varepsilon_{0}\right] \times D_{\varepsilon}\right) \cap\left(\mathbb{R}^{n} \backslash(\mathbf{E} \cup \mathcal{C})\right) ; \\
& \mathcal{C}(\varepsilon)=\mathcal{C} \cup \mathcal{T}(\varepsilon),
\end{aligned}
$$

where $\varepsilon_{0}>0$ is fixed sufficiently small in order that $\left[-\varepsilon_{0}, L+\varepsilon_{0}\right] \times\left\{0_{\mathbb{R}^{n-1}}\right\}$ crosses $\partial \mathcal{C}$ and $\partial \mathcal{B}$ at one point only. Then, the resonator is defined as,

$$
\Omega(\varepsilon):=\mathcal{C}(\varepsilon) \cup \mathbf{E} .
$$

For any domain $Q$, let $P_{Q}$ denote the Laplacian $-\Delta_{Q}$ with Dirichlet boundary conditions on $\partial Q$, and set $P_{\varepsilon}:=P_{\Omega_{\varepsilon}}$.

The resonances of $P_{\varepsilon}$ are defined as the eigenvalues of the operator obtained by performing a complex dilation with respect to $x$, for $|x|$ large.

Remark 2.1. As $\varepsilon \rightarrow 0_{+}$, the resonator $\Omega(\varepsilon)$ collapses to $\Omega_{0}:=\mathcal{C} \cup\left[0, M_{0}\right] \cup$ $\mathbf{E}$, where $M_{0}$ is the point $\left(L, 0_{\mathbb{R}^{n-1}}\right) \in \mathbb{R}^{n}$. In particular, for $\varepsilon=0$, the interior $\Omega_{0}=\mathcal{C} \cup \mathbf{E}$ of $\Omega_{0}$ is such that the resonances of $P_{\Omega_{0}}$ consist of the eigenvalues of $P_{\mathcal{C}}$ and the resonances of $P_{\mathbf{E}}$. It is well known that $P_{\mathbf{E}}$ has no imbedded eigenvalues, and thus its resonances (that are of course independent of $\varepsilon$ ) stay away from the real line. Moreover, by the results of $\left[\mathrm{Be},[\mathrm{HM}]\right.$, we know that the set of the resonances of $P_{\Omega_{0}}$ (that includes the eigenvalues of $P_{\mathcal{C}}$ ) is nothing but the limit set of those of $P_{\varepsilon}$ as $\varepsilon \rightarrow 0_{+}$.

We are interested in those resonances of $P_{\varepsilon}$ that are close to the eigenvalues of $P_{\mathcal{C}}$. Thus, let $\lambda_{0}>0$ be an eigenvalue of $P_{\mathcal{C}}$ with $u_{0}$ the corresponding normalized eigenfunction. As in [MN2], we assume,

$\lambda_{0}$ is the lowest eigenvalue of $P_{\mathcal{C}}$.

In the sequels, we denote by $u_{0}$ the corresponding positive normalized eigenfunction of $P_{\mathcal{C}}$.

By the arguments of $[\mathrm{Be}, \mathrm{HM}]$, we know that there is a unique resonance $\rho(\varepsilon) \in \mathbb{C}$ of $P_{\varepsilon}$ such that $\rho(\varepsilon) \rightarrow \lambda_{0}$ as $\varepsilon \rightarrow 0_{+}$. Furthermore, denoting by $\alpha_{0}$ the square root of the first eigenvalue of $-\Delta_{D_{1}}$, there is an eigenvalue $\lambda(\varepsilon)$ of $P_{\mathcal{C}(\varepsilon)}$ such that, for any $\delta>0$,

$$
|\rho(\varepsilon)-\lambda(\varepsilon)| \leq C_{\delta} e^{-2 \alpha_{0}(1-\delta) L / \varepsilon},
$$

for some $C_{\delta}>0$ and all sufficiently small $\varepsilon>0$. In particular, since $\lambda(\varepsilon) \in \mathbb{R}$, this gives

$$
|\operatorname{Im} \rho(\varepsilon)| \leq C_{\delta} e^{-2 \alpha_{0}(1-\delta) L / \varepsilon}
$$

We now state our main result. 
Theorem 2.2. Under Assumptions (2.1)-(2.3), for any $\delta>0$ there exists $C_{\delta}>0$ such that, for all $\varepsilon>0$ small enough, one has,

$$
|\operatorname{Im} \rho(\varepsilon)| \geq \frac{1}{C_{\delta}} e^{-2 \alpha_{0}(1+\delta) L / \varepsilon} .
$$

Remark 2.3. Gathering (2.5) and Theorem 2.2, we can reformulate the result as,

$$
\lim _{\varepsilon \rightarrow 0_{+}} \varepsilon \ln |\operatorname{Im} \rho(\varepsilon)|=-2 \alpha_{0} L .
$$

Remark 2.4. In the case where $\mathcal{C}$ is not connected (or $\mathcal{B}$ is not conttractible), the result is still valid under a straightforward additional condition on $\lambda_{0}$. Indeed, in this case the obstacle may contain several connected cavities (and they may admit $\lambda_{0}$ as eigenvalue, or not). However, in order that the result remains valid, it is sufficient to assume that the tube $\mathcal{T}(\varepsilon)$ connects the exterior $\mathbf{E}$ with one of the connected cavities admitting $\lambda_{0}$ as a simple eigenvalue.

\section{BACKGROUND PROPERTIES}

By definition, the resonance $\rho(\varepsilon)$ is an eigenvalue of the complex distorted operator,

$$
P_{\varepsilon}(\mu):=U_{\mu} P_{\varepsilon} U_{\mu}^{-1}
$$

where $\mu>0$ is a small parameter, and $U_{\mu}$ is a complex distortion of the form,

$$
U_{\mu} \varphi(x):=\varphi(x+i \mu f(x)),
$$

with $f \in C^{\infty}\left(\mathbb{R}^{n} ; \mathbb{R}^{n}\right), f=0$ near $\overline{\mathcal{B}}, f(x)=x$ for $|x|$ large enough. (In principle, $U_{\mu}$ may act on holomorphic functions $\varphi$ only, but the explicit form of $U_{\mu} P_{\varepsilon} U_{\mu}^{-1}$ as a second order differential operator allows to make act $P_{\varepsilon}(\mu)$ on $L^{2}\left(\mathbb{R}^{n}\right)$ with domain $H^{2}\left(\mathbb{R}^{n}\right)$; moreover, by Weyl Perturbation Theorem, the essential spectrum of $P_{\varepsilon}(\mu)$ is $e^{-2 i \theta} \mathbb{R}_{+}$, with $\theta=\arctan \mu$.)

It is well known that such eigenvalues do not depend on $\mu$ (see, e.g., [SZ, [HeM]), and that the corresponding eigenfunctions are of the form $U_{\mu} u_{\varepsilon}$ with $u_{\varepsilon}$ independent of $\mu$, smooth on $\mathbb{R}^{n}$ and analytic in a complex sector around E. In other words, $u_{\varepsilon}$ is a non trivial analytic solution of the equation $-\Delta u_{\varepsilon}=\rho(\varepsilon) u_{\varepsilon}$ in $\Omega(\varepsilon)$, such that $\left.u_{\varepsilon}\right|_{\partial \Omega(\varepsilon)}=0$ and, for all $\mu>0$ small enough, $U_{\mu} u_{\varepsilon}$ is well defined and is in $L^{2}(\Omega(\varepsilon)$ ) (in our context, this latter property will be taken as a definition of the fact that $u_{\varepsilon}$ is outgoing). Moreover, $u_{\varepsilon}$ can be normalized by setting, for some fixed $\mu>0$,

$$
\left\|U_{\mu} u_{\varepsilon}\right\|_{L^{2}(\Omega(\varepsilon))}=1 \text {. }
$$

In that case, we learn from [HM] (in particular Proposition 3.1 and formula (5.13)), that, for any $\delta>0$, and for any $R>0$ large enough, one has,

$$
\left\|u_{\varepsilon}\right\|_{L^{2}(\Omega(\varepsilon) \cap\{|x|<R\})}=1+\mathcal{O}\left(e^{\left(\delta-\alpha_{0}\right) L / \varepsilon}\right),
$$

and

$$
\left\|u_{\varepsilon}\right\|_{H^{1}(\mathbf{E} \cap\{|x|<R\})}=\mathcal{O}\left(e^{\left.\left(\delta-\alpha_{0}\right)\right) L / \varepsilon}\right) .
$$


Now, we take $R>0$ such that $\overline{\mathcal{B}} \subset\{|x|<R\}$. Using the equation $-\Delta u_{\varepsilon}=$ $\rho u_{\varepsilon}$ and Green's formula on the domain $\Omega(\varepsilon) \cap\{|x|<R\}$, and using polar coordinates $(r, \omega)$, we obtain,

$$
\operatorname{Im} \rho \int_{\Omega(\varepsilon) \cap\{|x|<R\}}\left|u_{\varepsilon}\right|^{2} d x=-\operatorname{Im} \int_{\mathbb{S}^{n-1}} \frac{\partial u_{\varepsilon}}{\partial r}(R, \omega) \bar{u}_{\varepsilon}(R, \omega) R^{n-1} d \sigma_{n-1}(\omega),
$$

(where $d \sigma_{n-1}(\omega)$ stands for the surface measure on $\mathbb{S}^{n-1}$ ), and thus, by (3.1), and for any $\delta>0$,

$$
\operatorname{Im} \rho=-\left(1+\mathcal{O}\left(e^{\left(\delta-2 \alpha_{0}\right) L / \varepsilon}\right)\right) \operatorname{Im} \int_{\mathbb{S}^{n-1}} \frac{\partial u_{\varepsilon}}{\partial r}(R, \omega) \bar{u}_{\varepsilon}(R, \omega) R^{n-1} d \sigma_{n-1}(\omega)
$$

where the $\mathcal{O}$ is locally uniform with respect to $R$.

Therefore, to prove our result, it is sufficient to obtain a lower bound on $\operatorname{Im} \int_{\mathbb{S}^{n-1}} \frac{\partial u_{\varepsilon}}{\partial r}(R, \omega) \bar{u}_{\varepsilon}(R, \omega) R^{n-1} d \sigma_{n-1}(\omega)$. Note that, by using (3.2), we immediately obtain (2.5).

Starting from this formula, the following proposition has been proved in MN2 (the proof is actually done in 2 dimensions only, but can be generalized easily to any dimension: see [MN2, Remark 4.6):

Proposition 3.1 (Martinez-Nédélec [MN2]). Let $R_{1}>R_{0}>0$ be fixed in such a way that $\overline{\mathcal{B}} \subset\left\{|x|<R_{0}\right\}$. Then, for any $C>0$, there exists a constant $C^{\prime}=C^{\prime}\left(R_{0}, R_{1}, C\right)>0$ such that, for all $\varepsilon>0$ small enough, one has,

$$
|\operatorname{Im} \rho| \geq \frac{1}{C^{\prime}}\left\|u_{\varepsilon}\right\|_{L^{2}\left(R_{0}<|x|<R_{1}\right)}^{2}-C^{\prime} e^{-C / \varepsilon} .
$$

Then, reasoning by contradiction as in MN2, we assume the existence of $\delta_{0}>0$ such that, along a sequence $\varepsilon \rightarrow 0_{+}$, one has

$$
|\operatorname{Im} \rho|=\mathcal{O}\left(e^{-2\left(\alpha_{0}+\delta_{0}\right) L / \varepsilon}\right) .
$$

Proposition 3.1 (added to standard Sobolev estimates) tells us that for any $R_{1}>R_{0}>0$ such that $\overline{\mathcal{B}} \subset\left\{|x|<R_{0}\right\}$, we have,

$$
\left\|u_{\varepsilon}\right\|_{H^{1}\left(R_{0}<|x|<R_{1}\right)}=\mathcal{O}\left(e^{-\left(\alpha_{0}+\delta_{0}\right) L / \varepsilon}\right) .
$$

Still following the procedure used in [MN2], we see that this estimate can be propagated up to the boundary of $\mathcal{B}$, away from an arbitrarily small neighborhood of $M_{0}:=\left(L, 0_{\mathbb{R}^{n-1}}\right)$ (this is done by means of Carleman inequalities up to the boundary [LR, LL]), and one obtains (see [MN2], Proposition 6.1),

Proposition 3.2 (Martinez-Nédélec [MN2]). Under the assumption (3.4), for any neighborhood $\mathcal{U}$ of $M_{0}$ and any compact set $K \subset \mathbb{R}^{n}$, there exists $\delta_{K}>0$ such that,

$$
\left\|u_{\varepsilon}\right\|_{H^{1}(\mathbf{E} \cap K \backslash \mathcal{U})}=\mathcal{O}\left(e^{-\left(\alpha_{0}+\delta_{K}\right) L / \varepsilon}\right),
$$

uniformly as $\varepsilon \rightarrow 0_{+}$.

From this point, the proof starts to differ completely from that of [MN2]. As a first step, we will improve this estimate by obtaining a control on the rate of decay when we come close to $M_{0}$. This will be achieved by using again Carleman estimates, but in the spirit of [KSU]. The final step will consist in 
propagating the estimate inside the thin tube, by using some "hand-made" Carleman estimate on such an $\varepsilon$-dependent domain. After that, the proof can be completed exactly as in MN2.

\section{Estimate NeAR $M_{0}$}

In order to extend the previous estimate close to $M_{0}$ with a good enough control on the rate of decay, we use the ideas of [KSU], in particular the notion of limiting Carleman weight. We will prove:

Proposition 4.1. Let $R>0$ and $s \in(0,1)$. Under assumption 3.4, there exists $\delta>0$ such that for all $\nu \in(0, R)$, one has,

$$
\left\|u_{\varepsilon}\right\|_{H^{1}\left(\left\{\nu<\left|x-M_{0}\right|<R\right\} \cap \boldsymbol{E}\right)}=\mathcal{O}\left(e^{-\left(\alpha_{0} L+\delta \nu^{s}\right) / \varepsilon}\right),
$$

uniformly for $\varepsilon>0$ small enough.

Remark 4.2. In Section 5, we will use this proposition with $s=\frac{1}{2}$.

Proof. We can assume $R>1$. Let $q \in(0,1)$ be a small parameter depending on $s$ and the geometry, to be specified later. It is sufficient to prove that there exists $\delta>0$ such that for all $k \geq 1$,

$$
\left\|u_{\varepsilon}\right\|_{H^{1}\left(\left\{q^{k}<\left|x-M_{0}\right|<R\right\} \cap \mathbf{E}\right)}=\mathcal{O}\left(e^{-\left(\alpha_{0} L+\delta q^{k s}\right) / \varepsilon}\right)
$$

uniformly for $\varepsilon>0$ small enough. We prove (4.1) by induction. Proposition 3.2 proves that there exists $\delta>0$ such that (4.1) holds with $k=1$.

We next assume (4.1) for some $k \geq 1$. We set,

$$
M_{1}:=\left(L-\frac{1}{8} q^{k+1}, 0_{\mathbb{R}^{n-1}}\right)=M_{0}-\left(\frac{1}{8} q^{k+1}, 0_{\mathbb{R}^{n-1}}\right) .
$$

For $x \in \mathbb{R}^{n}$, we define,

$$
\varphi(x):=\ln \left|x-M_{1}\right|
$$

and we denote by

$$
a(x, \xi):=\xi^{2}-(\nabla \varphi(x))^{2} \quad ; \quad b(x, \xi):=2 \nabla \varphi(x) \cdot \xi
$$

the real and imaginary parts of the principal symbol of the semiclassical operator $e^{-\varphi / h}\left(-h^{2} \Delta\right) e^{\varphi / h}$. Then, as observed in [DKSU], $\varphi$ is a limiting Carleman weight on $\mathbf{E}$ in the sense of [KSU], that is $|\nabla \varphi| \neq 0$ on $\mathbf{E}$, and $\{a, b\}=0$ on the set $\{a=b=0\}$ (where $\{a, b\}$ stands for the Poisson bracket between $a$ and $b$ ).

Moreover, thanks to (2.2), for $x \in \partial \mathcal{B}$ such that $\left|x-M_{0}\right| \leq \frac{1}{C} q^{k / 2}$, where $C>0$ is a constant given by the geometry of $\mathcal{B}$, we have

$$
\nabla \varphi(x) \cdot \vec{n}<0,
$$

where $\vec{n}$ stands for the outward pointing unit normal to $\mathbf{E}$ (or, equivalently, the inward pointing unit normal to $\mathcal{B}$ ). We can take $q$ small, so that $3 q^{k} \leq$ $\frac{1}{C} q^{k / 2}$ for any $k \geq 1$. Applying Proposition 3.2 of [KSU] with an open set of the form,

$$
V:=\mathbf{E} \cap\left\{\left|x-M_{1}\right|<3 q^{k}\right\},
$$


we see that, for any $v \in C^{\infty}(\bar{V}) \cap C_{0}^{\infty}\left(\overline{\mathbf{E}} \cap\left\{\left|x-M_{1}\right|<R\right\}\right)$ such that $\left.v\right|_{\partial \mathbf{E}}=0$, one has,

$$
\left\|e^{\varphi / h} v\right\|_{L^{2}(V)}^{2}+h^{2}\left\|e^{\varphi / h} \nabla v\right\|_{L^{2}(V)}^{2} \leq C_{0} h^{2}\left\|e^{\varphi / h} \Delta v\right\|_{L^{2}(V)}^{2},
$$

where the positive constant $C_{0}$ does not depend on $v$ and on $h>0$ small enough (but might depend on $k$ ).

Next, we apply this estimate with $v(x)=\chi\left(\left|x-M_{1}\right|\right) u_{\varepsilon}(x) \mathbb{1}_{\mathbf{E}}$, where

$$
\chi \in C_{0}^{\infty}\left(\left(\frac{q^{k+1}}{4}, 3 q^{k}\right) ;[0,1]\right), \quad \chi=1 \text { on }\left[\frac{q^{k+1}}{2}, 2 q^{k}\right],
$$

and $\mathbb{1}_{\mathbf{E}}$ stands for the characteristic function of $\mathbf{E}$. We also take $h:=\varepsilon / \mu_{k}$, where $\mu_{k}>0$ will be fixed later on. Setting $r:=\left|x-M_{1}\right|$, we obtain

$$
\left\|r^{\mu_{k} / \varepsilon} v\right\|^{2}+\varepsilon^{2} \mu_{k}^{-2}\left\|r^{\mu_{k} / \varepsilon} \nabla v\right\|^{2} \leq C_{0} \varepsilon^{2} \mu_{k}^{-2}\left\|r^{\mu_{k} / \varepsilon}([\Delta, \chi] u-\rho v)\right\|^{2},
$$

and thus, for $\varepsilon$ small enough,

$$
\left\|r^{\mu_{k} / \varepsilon} v\right\|^{2}+\varepsilon^{2} \mu_{k}^{-2}\left\|r^{\mu_{k} / \varepsilon} \nabla v\right\|^{2} \leq 2 C_{0} \varepsilon^{2} \mu_{k}^{-2}\left\|r^{\mu_{k} / \varepsilon}[\Delta, \chi] u\right\|^{2} .
$$

Now, we have

$$
\operatorname{Supp} \nabla \chi \subset\left\{r \in\left[\frac{q^{k+1}}{4}, \frac{q^{k+1}}{2}\right] \cup\left[2 q^{k}, 3 q^{k}\right]\right\} .
$$

Note that by the definition of $M_{1},\left|x-M_{1}\right| \geq 2 q^{k} \Longrightarrow\left|x-M_{0}\right| \geq q^{k}$. By the induction hypothesis (here and in the sequel $C$ denotes a large positive constant depending on $k$, that may change from line to line)

$$
\left\|r^{\mu_{k} / \varepsilon}[\Delta, \chi] u\right\|_{L^{2}\left(\left\{2 q^{k} \leq r \leq 3 q^{k}\right\} \cap \mathbf{E}\right)}^{2} \leq C\left(3 q^{k}\right)^{2 \mu_{k} / \varepsilon} e^{-\left(2 \alpha_{0} L+2 \delta q^{k s}\right) / \varepsilon} .
$$

By the a priori estimate (3.2) on $u$, for any $\delta^{\prime}>0$ there exists $C$ such that for small $\varepsilon>0$,

$$
\left\|r^{\mu_{k} / \varepsilon}[\Delta, \chi] u\right\|_{L^{2}\left(\left\{q^{k+1} / 4 \leq r \leq q^{k+1} / 2\right\} \cap \mathbf{E}\right)}^{2} \leq C\left(\frac{q^{k+1}}{2}\right)^{2 \mu_{k} / \varepsilon} e^{-\left(2 \alpha_{0} L-2 \delta^{\prime}\right) / \varepsilon} .
$$

We take $\delta^{\prime}=\mu_{k} \log \frac{6}{5}$ and obtain

$$
\left\|r^{\mu_{k} / \varepsilon}[\Delta, \chi] u\right\|_{L^{2}\left(\left\{q^{k+1} / 4 \leq r \leq q^{k+1} / 2\right\} \cap \mathbf{E}\right)}^{2} \leq C\left(\frac{3}{5} q^{k+1}\right)^{2 \mu_{k} / \varepsilon} e^{-2 \alpha_{0} L / \varepsilon} .
$$

Combining with (4.3) we obtain that for small $\varepsilon>0$,

$$
\begin{aligned}
\left(\frac{3}{4} q^{k+1}\right)^{2 \mu_{k} / \varepsilon}\|u\|_{H^{1}}^{2}\left(\left\{\frac{3}{4} q^{k+1} \leq r \leq 2 q^{k}\right\} \cap \mathbf{E}\right) & \\
& \leq C\left[\left(\frac{3}{5} q^{k+1}\right)^{2 \mu_{k} / \varepsilon}+\left(3 q^{k}\right)^{2 \mu_{k} / \varepsilon} e^{-\frac{2 \delta}{\varepsilon} q^{k s}}\right] e^{-2 \alpha_{0} L / \varepsilon},
\end{aligned}
$$

that is

$$
\begin{aligned}
&\left.\|u\|_{H^{1}\left(\left\{\frac{3}{4} q^{k+1} \leq r \leq 2 q^{k}\right\}\right.}^{2} \cap \mathbf{E}\right) \\
& \leq C\left[\left(\frac{4}{5}\right)^{2 \mu_{k} / \varepsilon}+\left(\frac{4}{q}\right)^{2 \mu_{k} / \varepsilon} e^{-\frac{2 \delta}{\varepsilon} q^{k s}}\right] e^{-2 \alpha_{0} L / \varepsilon} .
\end{aligned}
$$


At this point, we fix $\mu_{k}$ in such a way that one has,

$$
\left(\frac{4}{5}\right)^{2 \mu_{k} / \varepsilon}=\left(\frac{4}{q}\right)^{2 \mu_{k} / \varepsilon} e^{-\frac{2 \delta}{\varepsilon} q^{k s}}
$$

This gives $\mu_{k}:=\frac{\delta q^{k s}}{\log 5-\log q}$ (which is indeed $>0$ if $q$ is small), and (4.4) becomes,

$$
\|u\|_{H^{1}\left(\left\{\frac{3}{4} q^{k+1} \leq r \leq 2 q^{k}\right\} \cap \mathbf{E}\right)}^{2} \leq C e^{\frac{2 \delta q^{k s} \log \frac{4}{5}}{\varepsilon \log \frac{5}{q}}} e^{-2 \alpha_{0} L / \varepsilon} .
$$

We next choose $q$ so small that $\log \frac{5}{4} \geq q^{s} \log \frac{5}{q}$. This yields

$$
\frac{2 \delta q^{k s} \log \frac{4}{5}}{\varepsilon \log \frac{5}{q}} \leq-\frac{2 q^{(k+1) s} \delta}{\varepsilon} .
$$

From (4.5), we deduce

$$
\|u\|_{H^{1}\left(\left\{\frac{3}{4} q^{k+1} \leq r \leq 2 q^{k}\right\} \cap \mathbf{E}\right)}^{2} \leq C e^{-2 \delta q^{(k+1) s} / \varepsilon} e^{-2 \alpha_{0} L / \varepsilon} .
$$

Since $q^{k+1} \leq\left|x-M_{0}\right| \leq q^{k} \Longrightarrow \frac{3}{4} q^{k+1} \leq\left|x-M_{1}\right| \leq 2 q^{k}$, we obtain

$$
\|u\|_{H^{1}\left(\left\{q^{k+1} \leq\left|x-M_{0}\right| \leq q^{k}\right\} \cap \mathbf{E}\right)}^{2} \leq C e^{-2 \delta q^{(k+1) s} / \varepsilon} e^{-2 \alpha_{0} L / \varepsilon} .
$$

By the induction hypothesis, we can replace $q^{k+1} \leq\left|x-M_{0}\right| \leq q^{k}$ in the left-hand side of (4.6) by $q^{k+1} \leq\left|x-M_{0}\right| \leq R$, concluding the proof of (4.1) at $\operatorname{rank} k+1$.

\section{Estimate INSIDE THE NECK}

In this section we prove:

Proposition 5.1. Let $r_{0} \in(0, L)$, and assume (3.4). Then, there exists a small constant $\delta_{1}>0$ such that,

$$
\left\|u_{\varepsilon}\right\|_{H^{1}\left(\left[r_{0}, L\right] \times D_{\varepsilon}\right)}=\mathcal{O}\left(e^{-\left(\alpha_{0}+\delta_{1}\right) r_{0} / \varepsilon}\right),
$$

uniformly for $\varepsilon>0$ small enough.

The proof relies on the following Carleman inequality:

Lemma 5.2. Let $\eta_{0}>0$ be small enough. There exists $C>0$ such that for all $\alpha_{1}>0$ and $\varepsilon>0$, for all $v \in C^{\infty}(\overline{\Omega(\varepsilon)})$ verifying,

$$
\begin{gathered}
v_{\lceil\partial \Omega(\varepsilon)}=0 ; \\
\operatorname{Supp} v \subset\left\{x_{1}>0\right\} ; \\
\operatorname{Supp} v \cap \mathbf{E} \subset\left\{\left|x-M_{0}\right|<\eta_{0}\right\} ;
\end{gathered}
$$

one has,

$$
\frac{\alpha_{1}}{\varepsilon}\left\|e^{\alpha_{1} x_{1} / \varepsilon} v\right\|_{L^{2}(\Omega(\varepsilon))} \leq C\left\|e^{\alpha_{1} x_{1} / \varepsilon} \Delta v\right\|_{L^{2}(\Omega(\varepsilon))} .
$$


Proof of the lemma. We start with a general computation, valid for any open subset $\Omega$ of $\mathbb{R}^{n}$ with smooth boundary, and any function $v \in C^{\infty}(\bar{\Omega})$ satisfying Dirichlet boundary conditions. Let $\varphi \in C^{4}(\bar{\Omega})$ be real-valued. We will compute $\left\|e^{\varphi} \Delta v\right\|$.

We have $e^{\varphi} \Delta v=e^{\varphi} \Delta e^{-\varphi} w$, where $w=e^{\varphi} v$. Moreover, $-e^{\varphi} \Delta e^{-\varphi}=A+i B$, where $A$ and $B$ are the formally self-adjoint operators given by

$$
A w:=-\Delta w-|\nabla \varphi|^{2} w, \quad i B w:=2(\nabla \varphi) \cdot \nabla w+(\Delta \varphi) w .
$$

Then,

$$
\begin{aligned}
& \left\|e^{\varphi} \Delta v\right\|^{2} \\
& =\|(A+i B) w\|^{2}=\|A w\|^{2}+\|B w\|^{2}+2 \operatorname{Re} \int_{\Omega} A w \overline{i B w} d x .
\end{aligned}
$$

We claim:

$$
\begin{aligned}
& 2 \operatorname{Re} \int_{\Omega} A w \overline{i B w} d x=2 \int_{\Omega} \nabla\left(|\nabla \varphi|^{2}\right) \cdot \nabla \varphi|w|^{2} \\
& \quad+4 \int_{\Omega}(\nabla w)^{T} \varphi^{\prime \prime}(x) \nabla \bar{w}-\int_{\Omega} \Delta^{2} \varphi|w|^{2}-2 \int_{\partial \Omega} \partial_{n} \varphi\left|\partial_{n} w\right|^{2},
\end{aligned}
$$

where $\partial_{n}$ is the outward pointing normal unit at the boundary of $\Omega, \varphi^{\prime \prime}$ is the Hessian matrix of $\varphi$, and $(\nabla w)^{T}$ is the transpose of $\nabla w$.

Indeed, expanding $A w$ and $i B w$, we obtain:

$$
\begin{aligned}
2 \operatorname{Re} \int_{\Omega} A w \overline{i B w}= & -4 \operatorname{Re} \int_{\Omega} \Delta w \nabla \varphi \cdot \nabla \bar{w}-2 \operatorname{Re} \int_{\Omega} \Delta w \Delta \varphi \bar{w} \\
& -4 \operatorname{Re} \int_{\Omega}|\nabla \varphi|^{2} w \nabla \varphi \cdot \nabla \bar{w}-2 \int_{\Omega}|\nabla \varphi|^{2} \Delta \varphi|w|^{2} \\
= & I_{1}+I_{2}+I_{3}+I_{4} .
\end{aligned}
$$

We compute the terms $I_{j}, j=1,2,3$. Using the Green formula and the Dirichlet boundary condition for $w$, we obtain,

$$
I_{1}=4 \operatorname{Re} \int_{\Omega} \nabla w \cdot \nabla(\nabla \varphi \cdot \nabla \bar{w})-4 \operatorname{Re} \int_{\partial \Omega} \partial_{n} w \nabla \varphi \cdot \nabla \bar{w} .
$$

Since $w_{\uparrow \partial \Omega}=0$, one has $\nabla \varphi \cdot \nabla \bar{w}=\partial_{n} \varphi \cdot \partial_{n} \bar{w}$ on $\partial \Omega$. Moreover,

$$
\begin{aligned}
\operatorname{Re} \nabla w \cdot \nabla(\nabla \varphi \cdot \nabla \bar{w}) & =\sum_{1 \leq j, k \leq n} \frac{\partial w}{\partial x_{j}} \frac{\partial^{2} \varphi}{\partial x_{j} \partial x_{k}} \frac{\partial \bar{w}}{\partial x_{k}}+\frac{1}{2} \sum_{1 \leq j, k \leq n} \frac{\partial \varphi}{\partial x_{k}} \frac{\partial}{\partial x_{k}}\left|\frac{\partial w}{\partial x_{j}}\right|^{2} \\
& =(\nabla w)^{T} \varphi^{\prime \prime} \nabla \bar{w}+\frac{1}{2} \nabla \varphi \cdot \nabla\left(|\nabla w|^{2}\right) .
\end{aligned}
$$

Thus,

$$
I_{1}=4 \operatorname{Re} \int_{\Omega}(\nabla w)^{T} \varphi^{\prime \prime} \nabla \bar{w}-2 \int_{\Omega} \Delta \varphi|\nabla w|^{2}-2 \int_{\partial \Omega} \partial_{n} \varphi|\partial w|^{2} .
$$

On the other hand,

$$
I_{2}=2 \int_{\Omega}|\nabla w|^{2} \Delta \varphi+2 \operatorname{Re} \int_{\Omega} \nabla(\Delta \varphi) \cdot \nabla w \bar{w} .
$$


Since $\operatorname{Re} \nabla w \bar{w}=\frac{1}{2} \nabla|w|^{2}$, this yields

$$
I_{2}=2 \int_{\Omega}|\nabla w|^{2} \Delta \varphi-\int_{\Omega} \Delta^{2} \varphi|w|^{2} .
$$

Writing $I_{3}=-2 \int_{\Omega}|\nabla \varphi|^{2} \nabla \varphi \cdot \nabla|w|^{2}$, we obtain

$$
I_{3}=2 \int_{\Omega}|\nabla \varphi|^{2} \Delta \varphi|w|^{2}+2 \int_{\Omega} \nabla\left(|\nabla \varphi|^{2}\right) \cdot \nabla \varphi|w|^{2} .
$$

Combining (5.8), (5.9), (5.10) and (5.11), we deduce (5.7). When $\varphi$ depends only on the variable $x_{1}$, (5.7) becomes:

$$
\begin{aligned}
2 \operatorname{Re} \int_{\Omega} A w \overline{i B w}=4 \int_{\Omega} \varphi^{\prime \prime}\left|\partial_{x_{1}} w\right|^{2} & +4 \int_{\Omega} \varphi^{\prime \prime}\left(\varphi^{\prime}\right)^{2}|w|^{2} \\
& -\int_{\Omega} \varphi^{(4)}|w|^{2}-2 \int_{\partial \Omega} n_{1} \varphi^{\prime}\left|\partial_{n} w\right|^{2},
\end{aligned}
$$

where $n_{1}$ is the first coordinate of the outward pointing unit vector $\vec{n}$ at the boundary of $\Omega$.

We use (5.6), (5.12) with $\Omega=\Omega(\varepsilon), v$ satisfying the assumptions of the lemma, and

$$
\varphi\left(x_{1}\right)=\frac{\alpha_{1}}{\varepsilon} x_{1}+\frac{x_{1}^{2}}{2} .
$$

Note that $x_{1}$ is a limiting Carleman weight. The addition of the strictly convex term $\frac{x_{1}^{2}}{2}$ is in the spirit of [KSU]. However we cannot directly use the results of [KSU] since the domain of integration $\Omega(\varepsilon)$ depends on $\varepsilon$.

Note that $n_{1}=0$ at the boundary of the neck $[0, L] \times D_{\varepsilon}$. Chosing $\eta_{0}$ small, we have $n_{1} \varphi^{\prime}\left(x_{1}\right)=n_{1}\left(\alpha_{1} / \varepsilon+x_{1}\right) \leq 0$ on the intersection of the support of $w$ and $\partial \Omega(\varepsilon)$. Moreover $\varphi^{(4)}=0, \varphi^{\prime \prime}$ is nonnegative and

$$
\varphi^{\prime \prime}\left(x_{1}\right)\left(\varphi^{\prime}\left(x_{1}\right)\right)^{2}=\left(\alpha_{1} / \varepsilon+x_{1}\right)^{2} \geq \alpha_{1}^{2} / \varepsilon^{2} .
$$

Thus, we see that (5.12) implies:

$$
2 \operatorname{Re} \int_{\Omega(\varepsilon)} A w \overline{i B w} d x \geq 4 \frac{\alpha_{1}^{2}}{\varepsilon^{2}} \int_{\Omega(\varepsilon)}|v|^{2} e^{2 \alpha_{1} x_{1} / \varepsilon+x_{1}^{2}} .
$$

Hence, in view of (5.6),

$$
\left\|e^{\alpha_{1} x_{1} / \varepsilon+x_{1}^{2} / 2} \Delta v\right\| \geq \frac{2 \alpha_{1}}{\varepsilon}\left\|e^{\alpha_{1} x_{1} / \varepsilon+x_{1}^{2} / 2} v\right\| .
$$

Using that $x_{1}^{2} \leq\left(L+\eta_{0}\right)^{2}$ on the support of $v$, we obtain the conclusion of the lemma.

Proof of Proposition 5.1. We fix a small $\varepsilon_{0}>0$ and let $\chi \in C_{0}^{\infty}\left(\overline{\Omega\left(\varepsilon_{0}\right)}\right)$ such that

$$
\begin{cases}\chi(x)=0 & \text { if } x \in \mathcal{C} \text { or } x \in[0, \nu] \times D_{\varepsilon_{0}} \text { or }\left(x \in \mathbf{E} \text { and }\left|x-M_{0}\right| \geq \nu+\nu^{2}\right) \\ \chi(x)=1 & \text { if } x \in[2 \nu, L] \times D_{\varepsilon_{0}} \text { or }\left(x \in \mathbf{E} \text { and }\left|x-M_{0}\right| \leq \nu\right),\end{cases}
$$

where $\nu>0$ is a small parameter to be specified later. Of course, we can also consider $\chi$ as an element of $C_{0}^{\infty}(\overline{\Omega(\varepsilon)})$ for $0<\varepsilon<\varepsilon_{0}$. Using Lemma 
5.2 with $\alpha_{1}=\alpha_{0}+\nu^{3 / 4}$ and $v=\chi u$, we obtain, for small $\varepsilon>0$, denoting by $C_{\nu}$ a constant depending on $\nu$ and changing from line to line:

$$
\begin{aligned}
& \frac{1}{\varepsilon^{2}}\left\|\chi u e^{\left(\alpha_{0}+\nu^{3 / 4}\right) x_{1} / \varepsilon}\right\|^{2} \\
& \quad \leq C_{\nu}\left\|[\Delta, \chi] u e^{\left(\alpha_{0}+\nu^{3 / 4}\right) x_{1} / \varepsilon}\right\|^{2}+C_{\nu}|\rho(\varepsilon)|^{2}\left\|\chi u e^{\left(\alpha_{0}+\nu^{3 / 4}\right) x_{1} / \varepsilon}\right\|^{2} .
\end{aligned}
$$

Since $\rho(\varepsilon)$ is bounded uniformly as $\varepsilon \rightarrow 0$, the last term of (5.14) can be absorbed by its left-hand side for small $\varepsilon$ Moreover

$$
\left\|[\Delta, \chi] u e^{\left(\alpha_{0}+\nu^{3 / 4}\right) x_{1} / \varepsilon}\right\|^{2} \leq C_{\nu} \int_{\omega_{1} \cup \omega_{2}}\left(|\nabla u|^{2}+|u|^{2}\right) e^{2\left(\alpha_{0}+\nu^{3 / 4}\right) x_{1} / \varepsilon} d x
$$

where

$$
\begin{aligned}
& \omega_{1}:=\left\{x=\left(x_{1}, x^{\prime}\right): \nu \leq x_{1} \leq 2 \nu, x^{\prime} \in D_{\varepsilon}\right\} \\
& \omega_{2}:=\left\{x \in \mathbf{E}: \nu \leq\left|x-M_{0}\right| \leq \nu+\nu^{2}\right\} .
\end{aligned}
$$

Since $u$ remains locally bounded in $H^{1}(\Omega(\varepsilon))$ as $\varepsilon \rightarrow 0$, we can bound from above the integral on $\omega_{1}$ in (5.15) by $C_{\nu} e^{4\left(\alpha_{0}+\nu^{3 / 4}\right) \nu / \varepsilon}$. Using Proposition 4.1 with $s=\frac{1}{2}$, and chosing $\nu$ small enough, we bound from above the integral on $\omega_{2}$ by

$$
C_{\nu} e^{2\left(\alpha_{0}+\nu^{3 / 4}\right)\left(L+\nu+\nu^{2}\right) / \varepsilon} e^{-\left(2 \alpha_{0} L+2 \delta \nu^{1 / 2}\right) / \varepsilon} \leq C_{\nu} e^{-\delta \nu^{1 / 2} / \varepsilon},
$$

as $\varepsilon \rightarrow 0$. From (5.14), (5.15) and the estimates above,

$$
\frac{1}{\varepsilon^{2}}\|u\|_{L^{2}\left(\left[r_{0}, L\right] \times D_{\varepsilon}\right)}^{2} e^{2\left(\alpha_{0}+\nu^{3 / 4}\right) r_{0} / \varepsilon} \leq C_{\nu} e^{4\left(\alpha_{0}+\nu^{3 / 4}\right) \nu / \varepsilon} .
$$

Taking $\nu$ small enough, we have $\nu^{3 / 4} r_{0} \geq 4\left(\alpha_{0}+\nu^{3 / 4}\right) \nu$, and we deduce

$$
\|u\|_{L^{2}\left(\left[r_{0}, L\right] \times D_{\varepsilon}\right)}^{2} \leq C_{\nu} e^{-\left(2 \alpha_{0}+\nu^{3 / 4}\right) r_{0} / \varepsilon} .
$$

Using the equation $-\Delta \chi u=\rho \chi u+[\chi, \Delta] u$ with $\chi$ as before, and performing the scalar product with $\chi u$, we also deduce the same type of estimate for $\nabla u$, and this yields the conclusion of the proposition.

\section{Completion of the Proof of Theorem 2.2}

At this point, the completion of the proof is entirely taken from BHM1 (see also [BHM2, MN1, MN2]). For the sake of completeness, here we recall the main arguments (actually, in our setting they are a little bit simpler since we do not have to care too much about negative powers of $\varepsilon$ ). At first, using Assumption (2.3), we see that the eigenvalue $\lambda(\varepsilon)$ appearing in (2.4) is necessarily the first Dirichlet eigenvalue of $\mathcal{C}(\varepsilon)$, and, denoting by $v_{\varepsilon}$ the corresponding normalized positive eigenfunction, by [HM] we know that, for all $s \geq 0$ and $\delta>0$, one has,

$$
\left\|u_{\varepsilon}-v_{\varepsilon}\right\|_{H^{s}(\mathcal{C}(\varepsilon))}=\mathcal{O}\left(e^{-\left(\alpha_{0} L-\delta\right) / \varepsilon}\right),
$$

uniformly for $\varepsilon>0$ small enough. 
Then we use the explicit representation of $v=v_{\varepsilon}$ inside the tube in terms of the transversal modes,

$$
v=\sum_{k=0}^{+\infty} v_{k}\left(x_{1}\right) \varphi_{k}\left(x^{\prime} / \varepsilon\right)=\sum_{k=0}^{+\infty}\left(a_{k}^{+} e^{\theta_{k}\left(x_{1}-\mu\right) / \varepsilon}+a_{k}^{-} e^{-\theta_{k}\left(x_{1}-\mu\right) / \varepsilon}\right) \varphi_{k}\left(x^{\prime} / \varepsilon\right),
$$

where $a_{k}^{ \pm} \in \mathbb{R}, \theta_{k}:=\sqrt{\alpha_{k}^{2}-\varepsilon^{2} \lambda(\varepsilon)}, \alpha_{k}^{2}:=(k+1)$-th eigenvalue of $-\Delta_{D_{1}}$ $\left(\alpha_{k}>0\right), \varphi_{k}:=$ normalized $(k+1)$-th eigenfunction of $-\Delta_{D_{1}}, x^{\prime}:=$ $\left(x_{2}, \ldots, x_{n}\right)$, and $\mu:=C_{0} \varepsilon$ with $C_{0}>0$ sufficiently large such that $\{\mu\} \times$ $D_{\varepsilon} \cap \mathcal{C}=\emptyset$.

Using standard Sobolev estimates, we see that $\left|a_{k}^{ \pm}\right|=\mathcal{O}\left(\varepsilon^{-N_{0}}\right)$ for some $N_{0} \geq 0$ independent of $k$, and by the Dirichlet boundary condition at $x_{1}=$ $L+\varepsilon_{0}$, we deduce that $\left|a_{k}^{+}\right|=\mathcal{O}\left(\varepsilon^{-N_{0}} e^{-2 \alpha_{k} L / \varepsilon}\right)$ uniformly with respect to $k$. As a consequence, for any $x_{1} \in[\mu, L]$, we obtain,

$$
v_{k}\left(x_{1}\right)=v_{k}(\mu) e^{-\theta_{k}\left(x_{1}-\mu\right) / \varepsilon}+\mathcal{O}\left(\varepsilon^{-N_{0}} e^{-\alpha_{k} L / \varepsilon}\right) .
$$

Now, using Proposition 5.1, we see that, for any $r_{0} \in(0, L)$, the coefficients $a_{k}^{ \pm}$'s satisfy,

$$
\sum_{k \geq 0}\left(\left|a_{k}^{+}\right|^{2} e^{2 \alpha_{k} L / \varepsilon}+\left|a_{k}^{-}\right|^{2} e^{-2 \alpha_{k} r_{0} / \varepsilon}\right)=\mathcal{O}\left(e^{-2\left(\alpha_{0}+\delta_{1}\right) r_{0} / \varepsilon}\right) .
$$

In particular, $a_{0}^{ \pm}=\mathcal{O}\left(e^{-\delta_{1} / \varepsilon}\right)$, and thus,

$$
v_{0}(\mu)=\mathcal{O}\left(e^{-\delta_{1} / \varepsilon}\right) \text {. }
$$

Moreover, by Weyl's law and a general result of [Da], we know that,

$$
\alpha_{k} \sim k^{\frac{2}{n}} \quad ; \quad \sup _{D_{1}} \frac{\left|\varphi_{k}\right|}{\varphi_{0}}=\mathcal{O}\left(\left(\alpha_{k}\right)^{\frac{n}{2}}\right) \quad(k \rightarrow \infty) .
$$

Writing,

$$
v_{k}\left(x_{1}\right)=\varepsilon^{n-1} \int_{D_{1}} v\left(x_{1}, \varepsilon y\right) \varphi_{k}(y) d y=\varepsilon^{n-1} \int_{D_{1}} v\left(x_{1}, \varepsilon y\right) \varphi_{0}(y) \frac{\varphi_{k}(y)}{\varphi_{0}(y)} d y
$$

and taking advantage of the fact that $v$ and $\varphi_{0}$ are non negative, we deduce,

$$
v_{k}(\mu)=\mathcal{O}\left((k+1) e^{-\delta_{1} / \varepsilon}\right) .
$$

Inserting in (6.2), and taking $C>C_{0}$, we obtain,

$$
\|v\|_{L^{2}\left([C \varepsilon, L] \times D_{\varepsilon}\right)}=\mathcal{O}\left(e^{-\delta_{1} / \varepsilon}\right) .
$$

As a consequence, using the equation $-\Delta v=\lambda v$ and standard Sobolev inequalities, we obtain the existence of a constant $\delta_{2}>0$ such that, for $C>0$ large enough,

$$
\sup _{[C \varepsilon, L] \times D_{\varepsilon}}\left|v_{\varepsilon}\right|=\mathcal{O}\left(e^{-\delta_{2} / \varepsilon}\right) .
$$

Then, using the boundary Harnack inequality for non-negative solutions to parabolic equations (see, e.g., [FGS, BHM2]), we deduce,

$$
\sup _{\mathcal{C}(\varepsilon) \cap\{|x| \leq C \varepsilon\}}\left|v_{\varepsilon}\right|=\mathcal{O}\left(e^{-\delta_{2} / \varepsilon}\right),
$$


and thus, for any $C>0$ large enough,

$$
\left\|v_{\varepsilon}\right\|_{L^{2}(\mathcal{C}(\varepsilon) \cap\{|x| \leq C \varepsilon\})}=\mathcal{O}\left(e^{-\delta_{2} / \varepsilon}\right) .
$$

Taking $\chi_{\varepsilon} \in C_{0}^{\infty}(\{|x|<2 C \varepsilon\}), \chi_{\varepsilon}(x)=1$ for $|x| \leq C \varepsilon, \partial^{\alpha} \chi_{\varepsilon}=\mathcal{O}\left(\varepsilon^{-|\alpha|}\right)$, and using again the equation $-\Delta \chi_{\varepsilon} v=\lambda \chi_{\varepsilon} v+\left[\chi_{\varepsilon}, \Delta\right] v$, we see that a similar estimates holds for $|\nabla v|$. Then, fixing $C$ sufficiently large, and defining,

$$
w:=\left(1-\chi_{\varepsilon}(x)\right) v,
$$

we have $w \in\left(H^{2} \cap H_{0}^{1}\right)(\mathcal{C})$, and,

$$
-\Delta_{\mathcal{C}^{\prime}} w=\lambda(\varepsilon) w+r \quad ; \quad\|r\|_{L^{2}(\mathcal{C})}=\mathcal{O}\left(e^{-\delta_{2} / \varepsilon}\right) .
$$

Using the fact that $\lambda(\varepsilon) \rightarrow \lambda_{0}$ as $\varepsilon \rightarrow 0$, and that $\lambda_{0}$ is simple, we easily deduce (by writing the spectral projector on $u_{0}$ as a contour integral of the resolvent),

in $L^{2}(\mathcal{C})$. In particular,

$$
w=u_{0}+\mathcal{O}\left(e^{-\delta_{2} / \varepsilon}\right)
$$

$$
\left\|u_{0}\right\|_{L^{2}(\mathcal{C} \cap\{|x| \leq C \varepsilon\})}=\mathcal{O}\left(e^{-\delta_{2} / \varepsilon}\right) .
$$

However, since $u_{0}>0$ in $\mathcal{C}$, a well known result of Hopf (see, e.g., GiTr]) insures that $\partial_{n} u(0)<0$ (where $\partial_{n}$ stands for the outer normal derivative of $\mathcal{C})$. As a consequence, one necessarily has $u_{0}(x) \geq c d(x, \partial \mathcal{C})$ near 0 (with some $c>0$ ), and thus,

$$
\left\|u_{0}\right\|_{L^{2}(\mathcal{C} \cap\{|x| \leq C \varepsilon\})} \geq c \varepsilon^{1+\frac{n}{2}} .
$$

Estimate (6.3) is clearly in contradiction with this, and the proof is complete.

Remark 6.1. Actually, the arguments of [BHM1, BHM2] are a little bit more precise, and they lead to the lower bound (BHM2, Theorem 1.2),

$$
\left\|u_{\varepsilon}\right\|_{L^{2}\left(\left[r_{0}, L\right] \times D_{\varepsilon}\right)} \geq \frac{1}{C} \varepsilon^{1+\frac{n}{2}} e^{-\alpha_{0} r_{0} / \varepsilon},
$$

in contradiction with (5.1).

\section{REFERENCES}

[Be] J. T. Beale. Scattering frequencies of resonators. Comm. Pure Appl. Math., 26 (1973), 549-563.

[BHM1] R.M Brown, P.D. Hislop and A. Martinez. Lower Bounds on Eigenfunctions and the first Eigenvalue Gap. Differential Equations with Applications to Mathematical Physics W.F.Ames, E.M.Harell, J.V.Herod (Ed.), Mathematical and Science in Engineering, Vol. 192, Academic Press 22: 269-279, 1993.

[BHM2] R.M Brown, P.D. Hislop and A. Martinez. Lower bounds on the interaction between cavities connected by a thin tube. Duke Math. J., Vol. 73, No. 1 (1994), $163-176$

[Bu] N. Burq. Lower bounds for shape resonances widths of long range Schrödinger operators, Am. J. Math. , 124, 2002.

[Da] E.B. Davies. Heat Kernels and Spectral Theory Cambridge University Press , Cambridge, 1989.

[DKSU] D. Dos Santos Ferreira, C.E. Kenig, M. Salo, G. Uhlman. Limiting Carleman weights and anisotropic inverse problems. Invent. Math. 178 (2009), 119-171.

[FGS] E.B. Fabes, N. Garofalo, S. Salsa. A backward Harnack inequality and Fatou theorem for non-negative solutions of parabolic equations. Ill. J. Math. 30, 536-565, 1986. 
[Fe] C. A. Fernández. Spectral concentration for the Laplace operator in the exterior of a resonator. J. Math. Phys. 26 (1985), no. 3, 383-384.

[FL] C. Fernández, R. Lavine. Lower bounds for resonance width in potential and obstacle scattering. Comm. Math. Phys., 128, 263-284,1990.

[FLM] S. Fujiie, A. Lahamar-Benbernou, A. Martinez. Width of shape resonances for non globally analytic potentials. J. Math. Soc. Japan Volume 63, Number 1, 1-78, 2011.

[GiTr] D. Gilbarg, N.S. Trudinger. Elliptic Partial Differential Equations of Second Order. Springer-Verlag, Berlin, 1983.

[Ha] E. Harrel. General lower bounds for resonances in one dimension. Comm. Math. Phys. 86, 221-225,1982.

[HaSi] E. Harrel, B. Simon. The mathematical theory of resonances whose widths are exponentially small. Duke Math. 47 n.4, 845-902,1980.

[HS] B. Helffer, J. Sjöstrand. Résonances en limite semiclassique. Bull. Soc. Math. France, Mémoire 24/25, 1986.

[HeM] B. Helffer, A. Martinez. Comparaison entre les diverses notions de résonances. Helv. Phys. Acta, Vol.60, p.992-1003, 1987.

[HM] P. D. Hislop and A. Martinez. Scattering resonances of a Helmholtz resonator. Indiana Univ. Math. J. 40 no. 2, 767-788, 1991.

[KSU] C. E. Kenig, J. Sjöstrand, G. Uhlmann. The Calderón problem with partial data. Ann. of Math. (2) 165 (2007), no. 2, 567-591.

[LL] J. Le Rousseau and G. Lebeau. On Carleman estimates for elliptic and parabolic operators. Applications to unique continuation and control of parabolic equations. ESAIM: Control, Optimisation and Calculus of Variations, Volume 18 / Issue 03 / July 2012, pp 712-747

[LR] G. Lebeau and L. Robbiano. The Calderón problem with partial data. Annals of Mathematics 165 (2007), 567-5915.

[MN1] A. Martinez and L. Nedelec Optimal lower bound of the resonance widths for a Helmholtz tube-shaped resonator. J. Spectr. Theory ,2, 203-223, 2012.

[MN2] A. Martinez and L. Nedelec Optimal lower bound of the resonance widths for the Helmholtz resonator. Ann. Henri Poincaré, to appear. URL: http://arxiv.org/abs/1402.6493

[SZ] J. Sjöstrand and M. Zworski. Complex scaling and the distribution of scattering poles. J. Amer. Math. Soc., 4:729-769, 1991. 